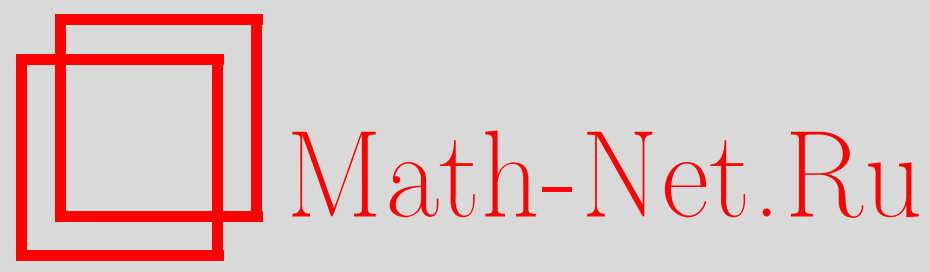

О. В. Бородин, А. В. Косточка, А. Распо, Э. Сопена, К оценке минимального числа цветов в ациклической $k$-сильной раскраске карт на поверхностях, Матем. заметки, 2002, том 72, выпуск 1, 35-37

DOI: https://doi.org/10.4213/mzm401

Использование Общероссийского математического портала Math-Net.Ru подразумевает, что вы прочитали и согласны с пользовательским соглашением http://www.mathnet.ru/rus/agreement

Параметры загрузки:

IP : 54.80 .73 .141

26 апреля 2023 г., $16: 48: 48$

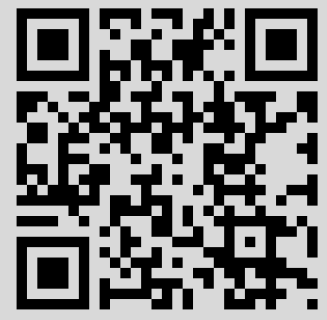




\title{
К ОЦЕНКЕ МИНИМАЛЬНОГО ЧИСЛА ЦВЕТОВ В АЦИКЛИЧЕСКОЙ $k$-СИЛЬНОЙ РАСКРАСКЕ КАРТ НА ПОВЕРХНОСТЯХ
}

\author{
О. В. Бородин, А. В. Косточка, А. Распо, Э. Сопена
}

\begin{abstract}
Раскраска вершин графа называется ациклической, если концы каждого ребра окрашены в разные цвета и нет двухцветных циклов. Пусть каждая грань ранга не более $k$, где $k \geqslant 4$, карта на поверхности $S^{N}$ заменена на клику с тем же множеством вершин. В [1] доказано, что тогда полученный псевдографф можно ациклически раскрасить в число цветов, линейно зависящее от $N$ и от $k$. В настоящей заметке эта оценка улучшена до $55(-N k)^{4 / 7}$ цветов при $k \geqslant 1$ и $-N \geqslant 5^{7} k^{4 / 3}$.

Библиография: 8 названий.
\end{abstract}

1. Введение. Раскраска вершин графа, вложенного в поверхность, назьвается $k$ сильной раскраской, если каждые две вершины на границе одной и той же грани ранга не более $k$ имеют разные цвета.

Раскраска вершин графа называется ациклической, если она правильная, т.е. конщы каждого ребра окрашены в разные цвета, и нет двухцветных циклов. Ациклическая раскраска имеет ряд приложений к другим задачам раскраски, (см. [2]-[6]).

Назовем картой псевдограф, вложенный в поверхность так, что не образуется граней рангов 1 и 2. Заметим, что петля порождает одноцветное ребро, а два кратных ребра двухцветный цикл. Будем считать раскраску вершин карты произвольной поверхности ациклической, если конщы каждого ребра-непетли е имеют разные цвета и нет двухцветных циклов длины больше 2. Алон, Мохар и Сандерс [7] доказали следующую оценку.

ТЕОРема 1. Каждый граф, вложимый в произвольную поверхность $S^{N}$, аииклически 50(2-N $)^{4 / 7}$-раскрашиваем, и эта оченка отличается от точной не более чем в $c(\log (3-N))^{1 / 7}$ раз.

При доказательстве они опирались на следующий результат Алона, МакДиармида и Рида [8].

Работа первого автора выполнена при частичной поддержке Российского фонда фундаментальных исследований, грант № 97-01-01075, и фонда INTAS, грант № 97-1001. Работа второго автора выполнена при частичной поддержке Российского фонда фундаментальных исследований, грант № 99-01-00581, и российско-голландского гранта NWO № 047-008-006. Работа третьего и четвертого авторов выполнена при частичной поддержке Совместного научного гранта НАТО № 97-1519. 
ТЕОРема 2 [8]. Каждый граф с максимальной степенью вершин не более $\Delta$ ачиклически $50 \Delta^{4 / 3}$-раскрашиваем.

В [1] рассматривается раскраска, являющаяся и ациклической, и $k$-цикловой. $\mathrm{A}$ именно, ациклически раскрашивается псевдограф $G^{(k)}$, получающийся заменой каждой грани ранга не более $k$ карты $G$ на клику с тем же множеством вершин. То есть подразумевается, что каждая грань ранга не более $k$ имеет все "невидимые диагонали". При $k=3$ такая раскраска совпадает с ациклической раскраской.

Основным результатом [1] является

ТЕОрема 3. Каждая карта на поверхности $S^{N}$ допускает ациклическую $k$-сильную раскраску в $c_{N} k+d_{N}$ иветов для любих $k \geqslant 4 u N \leqslant 2$, где $c_{N}=\max \{999$, $117-471 N\} u d_{N}=39-156 N$.

Обозначим через $F(k, N)$ наименьшее число цветов, достаточное для ациклической $k$-сильной раскраски картына поверхности $S^{N}$. По теореме $3 F(k, N)$ растет не быстрее чем линейно по $k$ и по $-N$. Легко показать, что $F(k, N)$ растет не медленнее чем линейно по $k$. А вот оценку по $-N$ можно улучшить. Цель данной заметки - доказать следующий факт.

TЕОРЕма 4. Пусть $k \geqslant 4 u$

$$
-N \geqslant 5^{7} k^{4 / 3} \text {. }
$$

Тогда кахсдая карта на поверхности $S^{N}$ допускает ациклическую $k$-сильную раскраску в $54(-N k)^{4 / 7}$ иветов.

Из теоремы 1 следует, что при любом фиксированном $k$ если эту оценку и можно улучшить, то не более чем в $c_{k}(\log (3-N))^{1 / 7}$ раз.

Доказательство теоремы 4 почти полностью повторяет доказательство теоремы 3 в [1], а в конце мы применяем теорему 2. Чтобы избежать больших повторений, мы будем опираться на текст [1]. В следующем разделе мы сначала укажем (небольшие) предлагаемые изменения в доказательстве теоремы 3 , а затем более детально опишем конец доказательства. Таким образом, следующий раздел надо читать, имея под рукой работу [1].

2. Доказательство теоремы 4. Повторяем доказательство теоремы 3 в [1] вплоть до (4) и пропускаем (5). Затем определяем $\operatorname{ch}(f)$, как в [1], для каждой грани $f$ и полагаем $\operatorname{ch}(v)=d(v)-4-f_{3^{*}}(v) / 3$ для каждой вершины $v$. Тогда вместо (6) имеем

$$
\sum_{v \in V_{3^{+}}} \operatorname{ch}(v)+\sum_{f \in F} \operatorname{ch}(f)<-4 N+1 .
$$

Далее отбрасываем лемму 4 и замечаем, что с новьм определением $\operatorname{ch}(v)$ лемма 5 справедлива уже при $d(v) \geqslant 6$. Затем полагаем $\varepsilon=\xi=0$ и перераспределяем вклады в точности как в [1]. Леммы 6-10 доказываются так же, как в [1], принимая во внимание, что у нас (согласно (1)) в запасе $54(-N k)^{4 / 7} \geqslant 54 \cdot 5^{4} k$ цветов. В частности, из этих лемм следует, что $\mathrm{ch}^{*}(x) \geqslant 0$ для любой грани или вершины $x$. Более того, к утверждению леммы 9 можно добавить, что $\mathrm{ch}^{*}(v) \geqslant d(v) / 4$ для любой вершины $v \mathrm{c} d(v) \geqslant 48$.

Пусть $L=\frac{17}{30}(-N)^{3 / 7} k^{-4 / 7}$ и $B-$ множество вершин степени больше $L$. Покажем, что

$$
|B| \leqslant\left\lfloor 30(-N k)^{4 / 7}\right\rfloor
$$


Действительно, если $|B|>30(-N k)^{4 / 7}$, то согласно (2) и новой версии леммы 9 (напомним, что $\left.L=\frac{17}{30}(-N)^{3 / 7} k^{-4 / 7} \geqslant \frac{17}{30}\left(5^{7} k^{4 / 3}\right)^{3 / 7} k^{-4 / 7}>48\right)$

$$
\begin{aligned}
-4 N+1 & >\sum_{v \in V_{3}+} \operatorname{ch}(v) \geqslant \sum_{v \in B} \operatorname{ch}^{*}(v) \geqslant \sum_{v \in B} \frac{d(v)}{4} \geqslant|B| \frac{L}{4} \\
& >30(-N)^{4 / 7} \frac{17}{4 \cdot 30}(-N)^{3 / 7} \geqslant \frac{17}{4}(-N) .
\end{aligned}
$$

Это невозможно при $-N \geqslant 4$, а из (1) следует, что $-N \geqslant 5^{7}$. Полученное противоречие доказьвает (3).

По определению множества $B$ максимальная степень в графе $G^{(k)}-B$ не превосходит $L k$. Следовательно, согласно теореме 2 вершины $G^{(k)}-B$ можно ациклически раскрасить в $50\left(\frac{17}{30}(-N k)^{3 / 7}\right)^{4 / 3} \leqslant 24(-N k)^{4 / 7}$ цветов. Окрасим каждую вершину в $B$ своим собственньм цветом. Всего будет затрачено не более $|B|+24(-N k)^{4 / 7}$ цветов. Согласно (3) эта сумма на больше $54(-N k)^{4 / 7}$. Теорема доказана.

\section{СПИСОК ЦИТИРОВАННОЙ ЛИТЕРАТУРЫ}

[1] Бородин О. В., Косточка А. В., Распо А., Сопена Е. Ацик лическая $k$-сильная раскраска карт на поверхностях // Матем. заметки. 2000. Т. 67. №1. С. 36-45.

[2] Alon N., Marshall T. H. Homomorphisms of edge-colored graphs and Coxeter groups // J. Algebraic Combinatorics. 1998. V. 2. P. 277-289.

[3] Grünbaum B. Acyclic colorings of planar graphs // Israel J. Math. 1973. V. 14. №3. P. 390-408.

[4] Jensen T. R., Toft B. Graph Coloring Problems. New York: Wiley, 1995.

[5] Nešetřil J., Raspaud A. Colored Homomorphisms of Colored Mixed Graphs. KAM Series № 98-376: Prague, 1998.

[6] Raspaud A., Sopena E. Good and semi-strong colorings of oriented planar graphs // Inform. Processing Letters. 1994. V. 51. P. 171-174.

[7] Alon N., Mohar B., Sanders D. P. On acyclic colorings of graphs on surfaces // Israel J. Math. 1996. V. 94. P. 273-283.

[8] Alon N., McDiarmid C., Reed B. Acyclic colorings of graphs // Random Struct. Algorithms. 1991. V. 2. P. 277-288.

(О.В. Бородин, А. В. Косточка) Институт математики СО РАН,

(А. Распо, Э. Сопена) Университет Бордо I, Франция

E-mail : brdnoleg@math.nsc.ru 